\title{
A variable-length window-wise parameter-dependent state of charge estimation by Kalman filters
}

\author{
Bataa Lkhagvasuren, Fellow, IEEE, Minkyu Kwak, Hong Sung Jin, Gyuwon Seo, Sungyool Bong and \\ Jaeyoung Lee
}

\begin{abstract}
This paper proposes a new window-wise state of charge (SOC) estimation algorithm based on Kalman filters (KF). In the first stage, the equivalent circuit model's parameters are estimated by a least square estimation window-wise, assuming a linear SOC and open-circuit voltage (OCV) relation. The algorithm accurately estimates the parameters and observes the changes that depend on SOC. Moreover, based on the estimated parameters, the OCV values are identified. In the next stage, window-wise linear Kalman filter(ES-LKF) without hysteresis and extended Kalman filter (ES-EKF) and sigma-point Kalman filter (ESSPKF) algorithm with hysteresis are executed to estimate SOC. Having fewer state equations and hysteresis parameters tuned up in an off-line way, the ES-EKF and ES-SPKF perform better than the algorithms considered in previous works. The algorithms are validated by experiments with real data obtained from lab tests.
\end{abstract}

Index Terms-Parameter estimation, State of charge, Battery, Kalman filter, Battery management system

\section{INTRODUCTION}

$\mathbf{T}$ HE battery is the most attractive energy storage device, because of its high energy density and portability. Portable utilities, electric vehicles, and industries use batteries as their main energy storage device. Scientists and engineers around the world are developing various battery technologies and the energy and power densities of the batteries are continuously advancing. Nowadays, lithium-ion batteries are being used extensively for their higher energy density, longer cycle life, and more environmental friendliness.

Electric vehicles (EV), Hybrid-electric vehicles (HEV), and Plug-in hybrid-electric vehicles (PHEV) are recent technologies to achieve efficient utilization of energy sources. All these electric-type vehicles employ the battery as their main or auxiliary energy storage system. To ensure safety and efficiency, the BMS monitors and controls the battery states and operations by measuring the current, voltage, and temperature. The SOC, which is the level of the remaining charge relative to the total

Manuscript received Month $\mathrm{xx}, 2 \mathrm{xxx}$; revised Month $\mathrm{xx}, \mathrm{xxxx}$; accepted Month $x, x x x x$. This research was supported by Basic Science Research Program through the NRF of Korea funded by the Ministry of Science, ICT and Future Planning (2017R1E1A1A03070061, 2020R1I1A3071769). (Corresponding author: Bataa Lkhagvasuren.)

Bataa Lkhagvasuren, Minkyu Kwak and Hong Sung Jin are with the department of Mathematics, Chonnam National University, Gwangju, Korea (e-mail: bat120@gmail.com). Gyuwon Seo, Sungyool Bong and Jaeyoung Lee are affiliated with School of Earth Science and Environmental Engineering, Gwangju Institute of Science and Technology (GIST), Gwangju, Republic of Korea. capacity, is a crucial parameter and is among the states that need to be monitored. Estimating SOC is the fundamental challenge for BMS because the parameter uncertainty and nonlinear dynamics of the battery make it a complex and difficult task.

About SOC estimation methods, one can find a large amount of literature. Here, we only describe the basic methods and the ones that are directly related to our work. Ampere-hour counting (Ah counting) [1] is the most basic and direct method to estimate SOC. In this method, SOC is calculated by integrating the loaded current to find how much charge is remained. Although, Ah counting is a useful theoretical method, it can not be used in a practice, because it suffers from the unknown initial SOC value and the accumulated uncertainty due to the integration process. Therefore, a proper recalibration must be incorporated into it and Ah counting method is often combined with other techniques to obtain better accuracy.

Another conventional approach is using look-up tables or SOC-OCV relation. This approach is used independently or in a combination of Ah counting [2], [3]. However, measuring OCV requires a long rest time, therefore, it is not used in online applications. Moreover, for the batteries which have a very flat SOC-OCV curve, this method is not applicable.

More sophisticated methods are the model-based approaches. These methods use mathematical equations to model the battery dynamics and calculate the SOC from the equations taking inputs such as voltage, current, and temperature. There are two types of battery models: electrochemical and electrical models. The electrochemical models use equations based on physical laws that govern the internal electrochemical processes in the battery. Although the electrochemical models are very accurate, the coupled partial differential equations make them very difficult to implement. An electrical model uses electrical circuit elements such as voltage source, resistors, and capacitors to describe battery dynamic behavior. It is ideal for system simulation and implementation in embedded microcontrollers [4]-[6]. For the details of above-mentioned methods and the other ones such as reduced-order models and machine learning-based approaches, we refer to excellent review papers [7], [8] and [9] and references therein.

The extended Kalman filter (EKF) is a widely used nonlinear KF and it linearizes the system dynamics efficiently. In a series of works [10], EKF was used as an electrical modelbased SOC estimation method. The main advantage of KF 
based methods to estimate SOC is that it can be performed very accurately and continuously during battery operation. The unscented KF and central difference KF methods [11]-[13], which are different forms of sigma-point KF (SPKF), address model nonlinearities more efficiently.

The battery model parameters change depending on the SOC level, operating temperature, and cycle number. The SOC estimation that takes into account these parameter changes should give better results. Therefore, online parameter estimation algorithms are desirable for that purpose. Rahimi-Eichi et al. [14]-[18] considered a moving window least-square filter for the piecewise linear model of $S O C-O C V$ curve. The advantage of this method is that it estimates simultaneously the model parameters as the parameters change during the battery operation and the method has a better stability.

By proposing a special discretization for battery's dynamics equations, the authors in [19] extracted the battery's electrical model parameters more efficiently and the initial value of SOC in each time window was found by a nonlinear optimization solver. In this paper, we introduce a refined version of the discretization used in [19] and execute KFs to estimate SOC. This combined estimation scheme, in which the initial value of OCV is computed window-wise and the parameter variability is taken into account, yields more accurate results.

The rest of this article is organized as follows. The section II introduces the battery model, the proposed discretization and parameter identification scheme. The section III discusses the use of KFs with and without the hysteresis for the identified parameters. In section IV, the experimental results with data obtained from lab tests were given to validate the proposed method.

\section{BATTERY MODEL AND PARAMETER IDENTIFICATION}

In this work, we assume the terminal voltage $V$ and current $I$ are known only, and we do not consider the temperature dependence and the battery capacity degradation. An electrical model (or equivalent circuit model) of a battery consists of a series resistance $R_{0}$ and parallel $R C$ components (Fig.1). The purpose of using an electrical model is to imitate or approximate the dynamic behavior of a battery by the dynamics of the electrical model. Theoretically, one can add as many $R C$ components as one wants. However, adding $R C$ components increases the order of the dynamical systems (order of differential equations) being modeled. Although higher-order electrical models can give accurate simulation results, computational complexity and numerical stability issues often degrade the estimation results. In the work [4], two $R C$ components are used to simulate the behavior of the Li-Po cells with reasonable accuracy. And each of the $R C$ components acts as a slow and fast change of the variable. But one $R C$ component model can yield good enough accuracy [10], [16], [17] for a shorter time window expressing faster transients.

The relation of $O C V$ and $S O C$ is, generally, nonlinear and contributes to the complexity of battery dynamics significantly. An approximation of the $S O C-O C V$ curve by simpler functions (linear, quadratic, sigmoid, etc.) is necessary to manipulate the model's underlying equations. In this paper, one $R C$ component model is considered as in the Fig.1, and the $S O C-O C V$ curve is represented by:

$$
O C V=f(S O C) \text {. }
$$

The terminal voltage $V$ is the sum of individual element voltages:

$$
V=O C V+V_{0}+V_{R C},
$$

where $V_{0}$ is the voltage across the resistor element $R_{0}$ and $V_{R C}$ is the voltage across the $R C$ component. The $V_{R C}$ follows the differential equation

$$
\dot{V}_{R C}=-\frac{1}{R C} V_{R C}+\frac{1}{C} I
$$

Combining (2) and (3) yields

$$
R C \dot{V}-R C R_{0} \dot{I}-\left(R_{0}+R\right) I+V=O C V+R C O \dot{C} V .
$$

The $S O C$, by definition, satisfies the equation

$$
S \dot{O} C=\frac{1}{Q} I,
$$

where $Q$ is the total capacity of the cell. For the time window $\left\{t_{k}\right\}, k=k_{0}, k_{0}+1, \ldots, k_{0}+n$, we approximate the $S O C$ $O C V$ curve by linear relation

$$
O C V=a S O C+b
$$

where $a$ and $b$ are constants. This approximation is reasonable one if the time window and range of variability for $S O C$ are short. Putting (6) into (4) gives

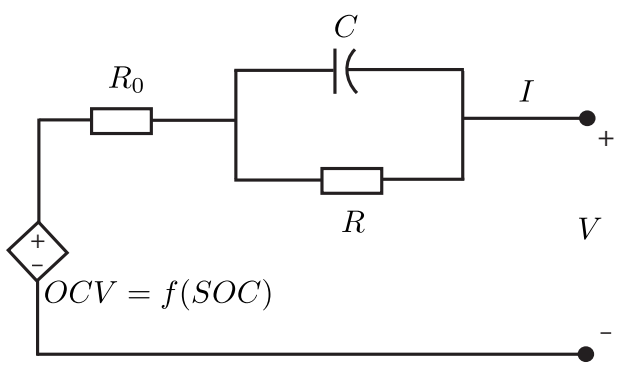

Fig. 1: Battery circuit model with one $R C$ branch

$R C \dot{V}-R C R_{0} \dot{I}-\left(R_{0}+R+\frac{a R C}{Q}\right) I+V=a S O C+b$.

In order to discretize the equations (7), we use the trapezoidal rule as follows:

$$
\begin{gathered}
f((k+1 / 2) T)=\frac{f[k+1]+f[k]}{2}, \\
\dot{f}((k+1 / 2) T)=\frac{f[k+1]-f[k]}{T}
\end{gathered}
$$

where $f[k]=f(k T)$. The bilinear transform, which is a transformation from continuous time system (in the Laplace domain) to discrete time system (in the Z-domain) uses the trapezoidal rule (8). Unlike the method used in [16], where 
the bilinear transform is applied to the second order version of (8) (one more differentiation), the trapezoidal rule is applied to (7) directly. In the next stage, the $S O C$ is expressed by coulomb counting method with the rectangle rule for integral:

$$
S O C[k]=S O C\left[k_{0}\right]+\frac{T}{Q}(J[k]-I[k])
$$

with $J[k]=\sum_{j=k_{0}}^{k} I[j]$ for $k=k_{0}, k_{0}+1, \ldots, k_{0}+n$.

In the work [19], trapezoidal rule for integral was used for coulomb counting. The reason that rectangle rule for integral was used in this paper is the equation (9) is compatible with the state equation in KF-based $S O C$ estimation. Another subtle effect is that (9) captures the instantaneous hysteresis more efficiently compared to trapezoidal rule (see section IV).

Using (8) for $V, I$ and computing $S O C$ by (9), the following discrete differential equations are obtained:

$$
V[k+1]=a_{1} V[k]+a_{2} I[k+1]+a_{3} I[k]+a_{4} J[k]+a_{5}
$$

where

$$
\begin{aligned}
a_{1} & =\frac{2 R C-T}{2 R C+T}, \\
a_{2} & =\frac{2 R C R_{0}+T\left(R_{0}+R+a R C / Q\right)}{2 R C+T}, \\
a_{3} & =\frac{-2 R C R_{0}+T\left(R_{0}+R+a R C / Q\right)-a T^{2} / Q}{2 R C+T}, \\
a_{4} & =\frac{2 a T^{2}}{Q(2 R C+T)}, \\
a_{5} & =\frac{2 T O C V\left[k_{0}\right]}{2 R C+T} .
\end{aligned}
$$

The values $V[k+1], V[k], I[k+1], I[k], J[k]$ are known (measured) values within the current time window, the system (10) and (11) can be solved in a least square sense. Therefore, the parameters $a, R_{0}, R, C$ and the value $O C V\left[k_{0}\right]$ are estimated directly. In the next stage, if we are to ignore the hysteresis effect, the value $S O C\left[k_{0}\right]$ and the parameter $b$ can be determined by

$$
S O C\left[k_{0}\right]=f^{-1}\left(O C V\left[k_{0}\right]\right), b=O C V\left[k_{0}\right]-a S O C\left[k_{0}\right] .
$$

The estimation algorithm purposed in [16] was able to determine only 4 parameters $a, R_{0}, R, C$ in the first stage and a look-up table of piece-wise linearization of $S O C-O C V$ curve was used to determine $b$. However, the estimation scheme (10)(12) determines not only the parameters $a, b, R_{0}, R, C$, but also the initial values of $S O C\left[k_{0}\right]$ and $O C V\left[k_{0}\right]$. Moreover, the linearization (6) of $S O C-O C V$ curve was performed within the time window, while the linearization purposed in [16] was done statically (not depending on time). Therefore, our estimation scheme has a better adaptation, and extensive numerical testing results are reported in [19]. Since the initial value $O C V\left[k_{0}\right]$ was determined, the circuit voltage $V_{R C}$ can be computed by

$$
\begin{gathered}
V_{R C}[k+1]=e^{-\frac{T}{R C}} V_{R C}[k]-R\left(e^{-\frac{T}{R C}}-1\right) I[k], \\
V_{R C}\left[k_{0}\right]=V\left[k_{0}\right]-O C V\left[k_{0}\right]-R_{0} I\left[k_{0}\right]
\end{gathered}
$$

within time window. Consequently, the $O C V[k]$ values are determined by

$$
O C V[k]=V[k]-V_{R C}[k]-R_{0} I[k] .
$$

In the next section, we purpose KF-based $S O C$ estimation algoritm that use the values (15).

\section{SOC ESTIMATION BY KFS}

Gaussian sequential probabilistic inference scheme is used for the following general model:

$$
\begin{aligned}
x_{k+1} & =f\left(x_{k}, u_{k}, w_{k}\right), \\
y_{k} & =g\left(x_{k}, u_{k}, v_{k}\right)
\end{aligned}
$$

where $w_{k}$ and $v_{k}$ Gaussian noise processes with means $\bar{w}$ and $\bar{v}$ and covariance matrices $\Sigma_{w}$ and $\Sigma_{v}$ respectively. The following is the summary of general Gaussian sequential probabilistic inference scheme, where Plett's notations were used from [11].

\section{Definition}

$$
\begin{array}{ll}
\tilde{x}_{k}^{-}=x_{k}-\widehat{x}_{k}^{-}, & \tilde{y}_{k}=y_{k}-\widehat{y}_{k} \\
\Sigma_{\tilde{y}, k}=E\left[\left(\tilde{y}_{k}\right)\left(\tilde{y}_{k}\right)^{T}\right] & \Sigma_{\tilde{x} \tilde{y}, k}^{-}=E\left[\left(\tilde{x}_{k}^{-}\right)\left(\tilde{y}_{k}\right)^{T}\right]
\end{array}
$$$$
Y_{k}=\left\{y_{0}, y_{1}, \ldots, y_{k}\right\}
$$

\section{Initialization}

For $k=0$, set

$$
\begin{aligned}
& \hat{x}_{0}^{+}=E\left[x_{0}\right] \\
& \Sigma_{\tilde{x}, 0}^{+}=E\left[\left(x_{0}-\hat{x}_{0}^{+}\right)\left(x_{0}-\hat{x}_{0}^{+}\right)^{t}\right]
\end{aligned}
$$

\section{Predict}

State estimate

Covariance

\section{Update}

Output estimate $\quad \hat{y}_{k}=E\left[g\left(x_{k}, u_{k}, v_{k}\right) \mid Y_{k-1}\right]$

Optimal Kalman gain $L_{k}=\Sigma_{\tilde{x} \tilde{y}, k}^{-} \Sigma_{\tilde{y}, k}^{-1}$

State estimation $\quad \hat{x}_{k}^{+}=\hat{x}_{k}^{-}+L_{k}\left[y_{k}-\widehat{y}_{k}\right]$

Error covariance $\quad \Sigma_{\tilde{x}, k}^{+}=\Sigma_{\tilde{x}, k}^{-}-L_{k} \Sigma_{\tilde{y}, k} L_{k}^{T}$

When the dynamics of the system being modeled is linear, i.e., the functions $f$ and $g$ are linear, the above scheme called linear KF, and it is exact minimum mean-square-error state estimator. If the system dynamics is nonlinear, other variants of KF are used. The EKF linearizes the model at each time point, and it performs well when the system nonlinearities are not high. The SPKF (or unscented KF) linearizes the model statistically at each point in time, and it tends to give reasonable estimates even if nonlinearities are high.

In the first phase of purposed $S O C$ estimation method, the parameters $a, b, R_{0}, R, C$ and the $O C V[k]$ values are estimated by (10)-(15). In the next phase, two types of KF (linear and nonlinear) are purposed depending on the influence of hysteresis effect. If the hysteresis effect is neglected, the following simple linear model can be used window-wise to estimate $S O C$ :

$$
\begin{aligned}
S O C[k+1] & =S O C[k]+\frac{T}{Q} I[k]+w[k], \\
O C V[k] & =a S O C[k]+b+v[k],
\end{aligned}
$$

where $w[k]$ and $v[k]$ are process and sensor noises with Gaussian distributions. Let us denote this combined estimation scheme (10)-(15) and (17a)-(17b) by ES-LKF. 
If we are to consider hysteresis effect significantly, the following hysteresis model can be used:

$$
O C V=f(S O C)+M_{0} s[k]+M h[k],
$$

where $f(S O C)$ denotes the average of main charge curve and discharge curve. The hysteresis modeling (18) is introduced in [20] as a part of enhanced self correcting model (ESC). The hysteresis effect is very complicated process and there is certain difficulty for modeling it, we refer to [20] and [21] for detailed discussions. The parameters $M_{0}, M$ and $\eta$ should be estimated in an offline way for measured values of $O C V-f(S O C)$. Therefore, the dynamic state-space model of a battery is written as

$$
\begin{gathered}
S O C[k+1]=S O C[k]+\frac{T}{Q} I[k]+w[k], \\
h[k+1]=e^{-\eta I[k] T / Q} h[k]+\operatorname{sign}(I[k])\left(1-e^{-\eta I[k] T / Q}\right), \\
O C V[k]=f(S O C[k])+M_{0} s[k]+M h[k]+v[k] .
\end{gathered}
$$

The equations (19a) and (19b) are the state equations and (19c) is the output equation for KF algorithm. Note that $V_{R C}[k]$ is not included as one of battery states compared to the original model introduced in [20], since it is determined already.

\section{TEST WITH LITHIUM-ION "E2" CELL}

In this section, the results of experimental tests of the proposed method are given for real data obtained from [20]. We compared the performance of the proposed method with that of EKF and SPKF reported in [20], where the parameters were extracted without considering $S O C$ dependence. The tests are executed for lithium-ion "E2" cell with the temperature $T=5^{\circ} \mathrm{C}$ and $T=25^{\circ} \mathrm{C}$. The battery's current and terminal voltage is displayed in Fig.2. This data is obtained by repeatedly exercising the "urban dynamometer drive schedule" (UDDS) profile. There are around 720 seconds of rest time after each UDDS profile exercise. Fig.4 shows the charge and discharge curves at the temperatures $T=5^{\circ} \mathrm{C}$ and $T=25^{\circ} \mathrm{C}$.

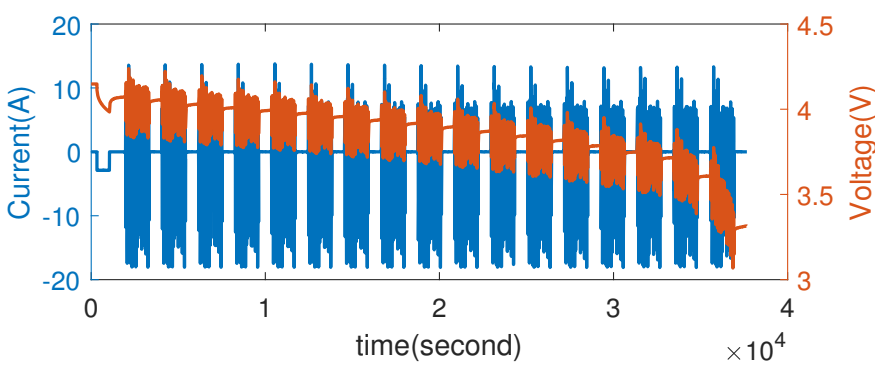

Fig. 2: Current and voltage of dynamic test data of the UDDS pattern $\left(T=25^{\circ} \mathrm{C}\right)$

In order to estimate $M, M_{0}$ and $\eta$ in (18) in an offline way, the following procedure was performed. Starting from the end of rest time, the parameter estimation algorithm (10), (11), (13), (14) and (15) is executed with 800 as the size of time window and $R_{0}, R, C, O C V$ are estimated. After determining $O C V-f(S O C)$ for known $S O C$, the parameters $\widehat{M}, \widehat{M}_{0}$

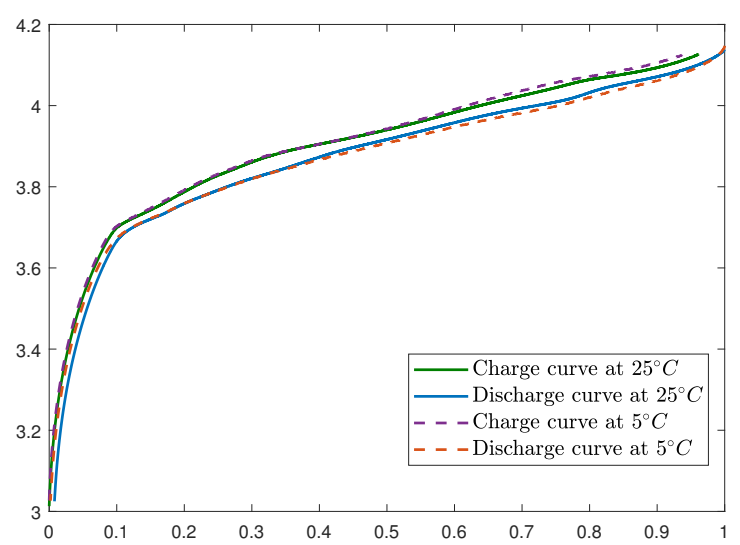

Fig. 3

Fig. 4: $S O C-O C V$ relation at $T=5^{\circ} \mathrm{C}$ and $T=25^{\circ} \mathrm{C}$

TABLE I: The parameters reported in [20].

\begin{tabular}{llllll}
\hline & $R 0$ & $R$ & $C$ & $M$ & $M_{0}$ \\
\hline$T=25^{\circ} C$ & 0.0112 & 0.0025 & 958.4886 & 0.0443 & 0.0025 \\
$T=55^{\circ} \mathrm{C}$ & 0.0313 & 0.0071 & 254.9203 & 0.0805 & 0 \\
\hline
\end{tabular}

and $\widehat{\eta}$ are estimated for different level of $S O C$ (4,5,6-th columns in Table II). This task is performed with the help of lsqcurvefit tool in Matlab by splitting the linear and nonlinear parts. 2, 3-d columns of Table II show the values of $M, M_{0}$ estimated linearly for $\eta_{25}=39.6, \eta_{5}=64.4$, which are taken as the averages of estimated values $\hat{\eta}$ for each temperature. We observe that for the case $T=25^{\circ} \mathrm{C}$ the instantaneous hysteresis parameter $M_{0}$ is estimated significantly compared with that reported in [19]. This is because the rectangle rule for integral used in (9) captures the instantaneous hysteresis more efficiently, whereas the trapezoidal rule for integral was used instead in [19]. Another effect observed in Table II is hysteresis level for $T=5^{\circ} \mathrm{C}$ is higher than for $T=25^{\circ} \mathrm{C}$, which is expected, since the battery dynamics is more severe in cooler temperatures. Let us denote the EKF and SPKF based on (10), (11), (13), (14), (15), (19a) and (19b) by ES-EKF and ES-SPKF respectively. Fig.5 shows the estimated parameters and time window sizes with excitation level 790. The values of $R_{0}, R$ and $C$ reported in [20] are held constant and shown in Table I. The noise covariances $\Sigma_{w}=0.2$ and $\Sigma_{v}=0.2$, which are used for SPKF and EKF in [20], are also used for ESSPKF in order to compare their performances. However, the measurement equation of ES-LKF is quite different in the form from that of ES-SPKF, SPKF and EKF. Thus, we found the value $\Sigma_{v}=1.6207 e-4$ by trial-and-error. The performances of the methods ES-Ah, ES-LKF, ES-SPKF, SPKF and EKF are compared in Table III and Figure 6,7. As expected the estimation results for ES-Ah and ES-LKF are similar for the reason mentioned in the previous section. Moreover, because of the hysteresis effect, the initial value $S O C\left[k_{0}\right]$ and $b$ determined by (12) are very unstable. The uncertainty in the measurement equation is so big that ES-LKF suffers from large error bound and very unstable SOC estimation as depicted 
TABLE II: Offline estimation of $M_{0}, M$ and $\eta$ for $T=5^{\circ} C$.

\begin{tabular}{|c|c|c|c|c|c|}
\hline$S O C$ & $M$ & $M_{0}$ & $\widehat{M}$ & $\widehat{M_{0}}$ & $\widehat{\eta}$ \\
\hline 0.887 & 0.040 & 0.0034 & 0.042 & 0.0042 & 125.5 \\
\hline 0.840 & 0.052 & 0.0021 & 0.052 & 0.0022 & 70.2 \\
\hline 0.793 & 0.047 & 0.0022 & 0.047 & 0.0021 & 59.1 \\
\hline 0.746 & 0.046 & 0.0021 & 0.045 & 0.0022 & 74.5 \\
\hline 0.699 & 0.049 & 0.0021 & 0.049 & 0.0022 & 70.2 \\
\hline 0.652 & 0.053 & 0.0023 & 0.054 & 0.0022 & 57.8 \\
\hline 0.604 & 0.057 & 0.0024 & 0.057 & 0.0023 & 57.0 \\
\hline 0.557 & 0.059 & 0.0024 & 0.059 & 0.0023 & 57.0 \\
\hline 0.510 & 0.062 & 0.0026 & 0.062 & 0.0024 & 57.0 \\
\hline 0.463 & 0.068 & 0.0025 & 0.069 & 0.0023 & 57.0 \\
\hline 0.416 & 0.077 & 0.0027 & 0.077 & 0.0025 & 57.0 \\
\hline 0.369 & 0.091 & 0.0032 & 0.091 & 0.0029 & 57.0 \\
\hline 0.322 & 0.100 & 0.0035 & 0.100 & 0.0032 & 57.0 \\
\hline 0.275 & 0.096 & 0.0036 & 0.097 & 0.0033 & 57.0 \\
\hline 0.228 & 0.090 & 0.0035 & 0.090 & 0.0032 & 57.0 \\
\hline 0.181 & 0.078 & 0.0028 & 0.078 & 0.0026 & 57.0 \\
\hline 0.134 & 0.115 & 0.0022 & 0.115 & 0.0026 & 74.5 \\
\hline 0.087 & 0.228 & 0.0053 & 0.229 & 0.0046 & 57.0 \\
\hline
\end{tabular}
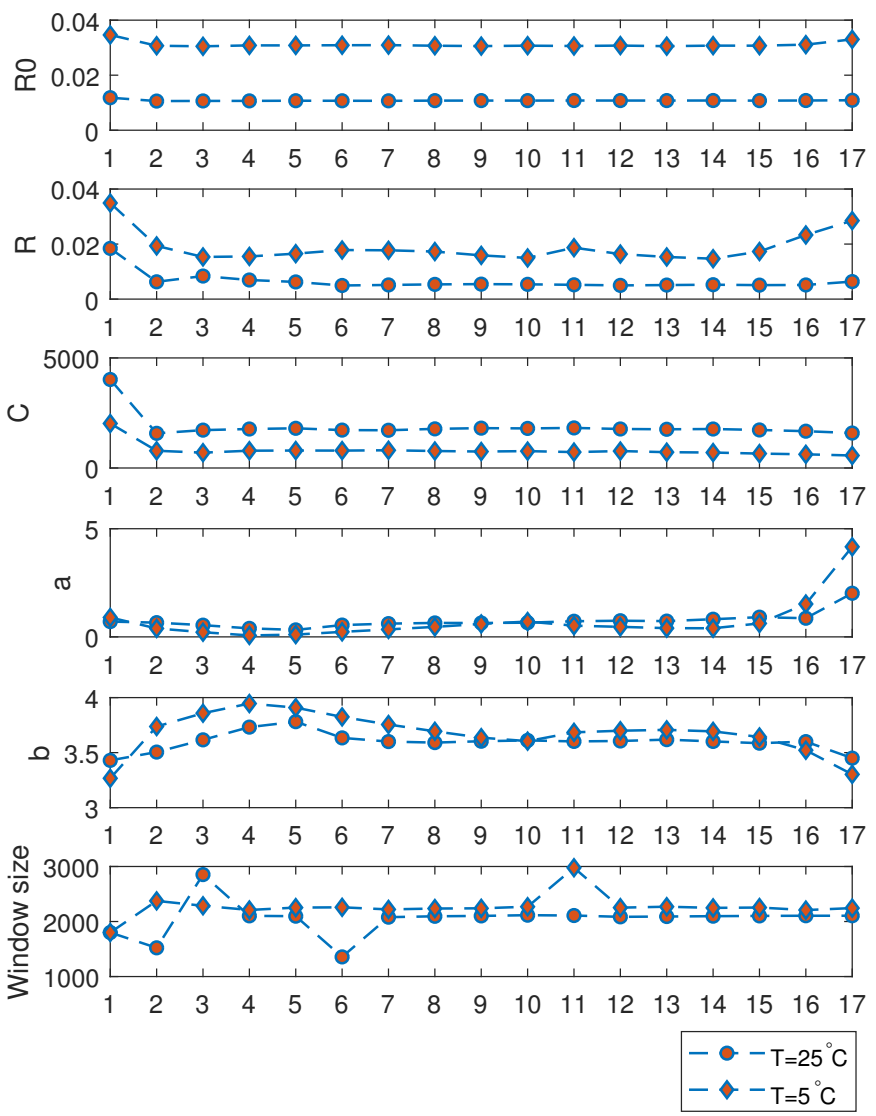

Fig. 5: Estimated parameters and time window sizes for $T=$ $25^{\circ} \mathrm{C}$ and $T=5^{\circ} \mathrm{C}$ with excitation level 790 .

in Fig.7. The method ES-SPKF outperforms all the others. This improvement can be explained by three ideas proposed in this work. First, the nonlinearity in battery dynamics localized efficiently and the parameters are extracted online as in Fig.5. Second, the initial value $O C V\left[k_{0}\right]$ is estimated along with other parameters and it enables us to calculate all $O C V[k]$. This process lets us to use fewer state equations (19a) and
TABLE III: RMS errors (in percents).

\begin{tabular}{lrr}
\hline Methods & $T=25^{\circ} \mathrm{C}$ & $T=5^{\circ} \mathrm{C}$ \\
\hline ES-Ah & $2.4981 \%$ & $7.9004 \%$ \\
\hline ES-LKF & $2.6363 \%$ & $8.6335 \%$ \\
EOB & $4.9495 \%$ & $36.9960 \%$ \\
\hline EKF & $0.5104 \%$ & $1.5252 \%$ \\
EOB & $2.0286 \%$ & $35.9171 \%$ \\
\hline ES-EKF & $0.1580 \%$ & $0.5696 \%$ \\
EOB & $0 \%$ & $0 \%$ \\
\hline SPKF & $0.5341 \%$ & $0.8368 \%$ \\
EOB & $3.6723 \%$ & $10.5274 \%$ \\
\hline ES-SPKF & $0.1569 \%$ & $0.2650 \%$ \\
EOB & $0 \%$ & $0.2783 \%$ \\
\hline & &
\end{tabular}
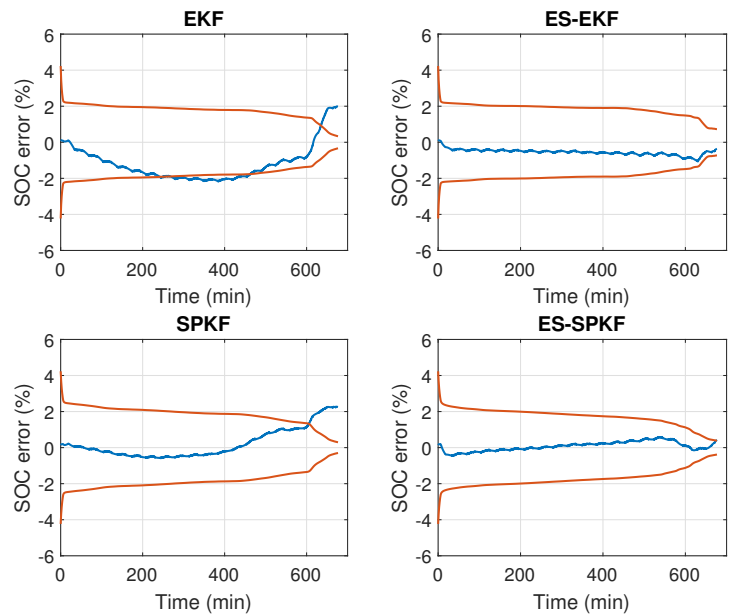

Fig. 6: Error bounds for $T=5^{\circ} \mathrm{C}$

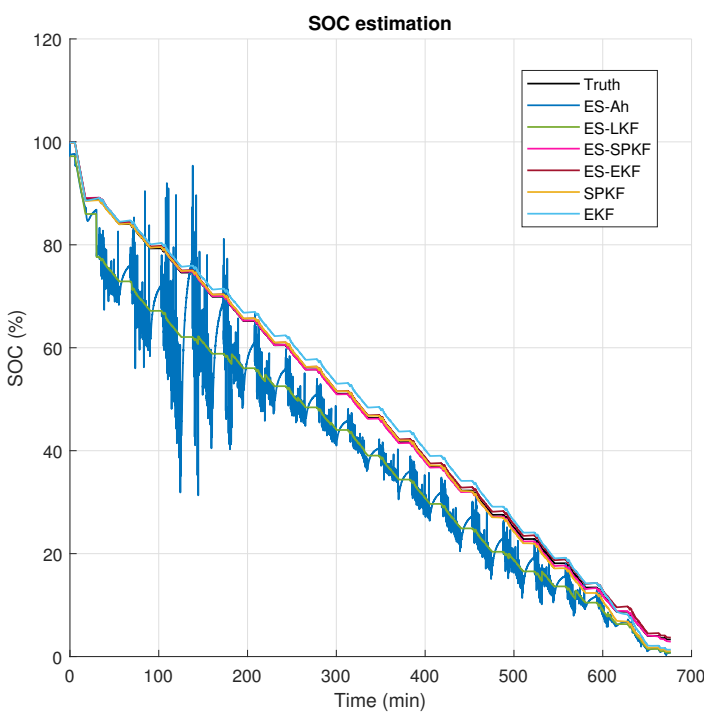

Fig. 7: $S O C$ estimation for $T=5^{\circ} \mathrm{C}$ 
(19b) in KF alforithm. Third, the hysteresis parameters are extracted in a way such that they depend on $S O C$ as in Table II.

\section{TEST WITH NMC532/GRAPHITE POUCH CELL}

This section presents the test results for NMC532/Graphite pouch cell. A pouch cell consists of a graphite anode, and

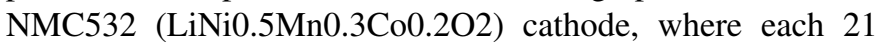
and 20 two-side coated anode and cathodes are stacked as zig-zag stacking method as in Fig.8. This stacked anodes and cathodes are wrapped by $\mathrm{Al}$ pouch film with liquid electrolyte. The cell operating voltage range is $3.0-4.25 \mathrm{~V}$ and the maximum continuous charge/discharge current is $0.7 \mathrm{C}$.

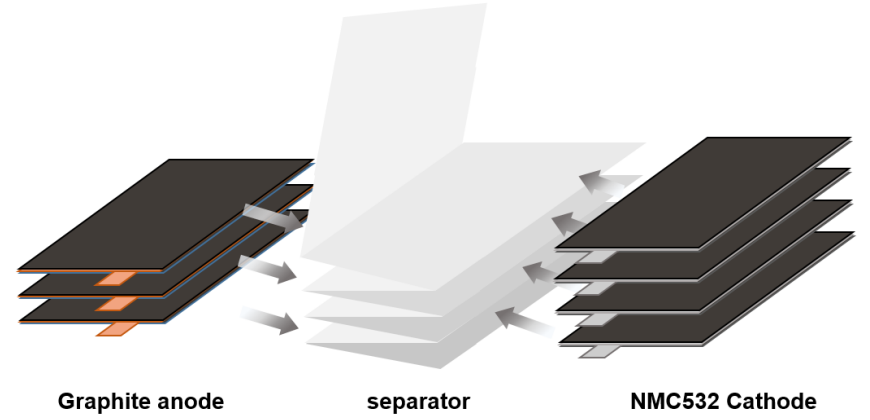

Fig. 8: The structure of NMC532/Graphite pouch cell.
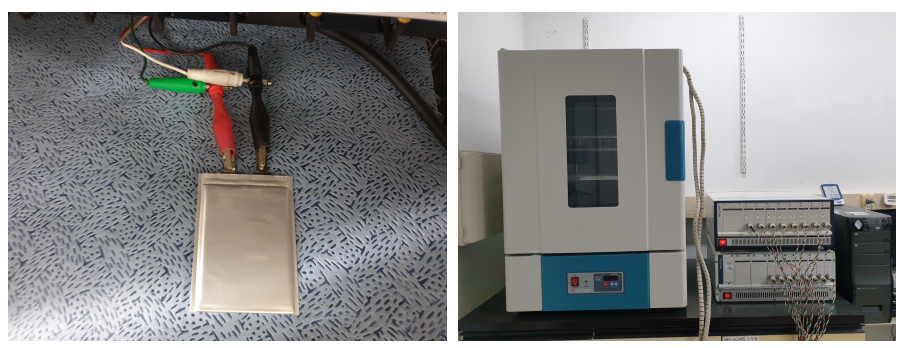

(a) The NMC532/Graphite pouch cell and the incubator(right) for keeping the cell under $25^{\circ} \mathrm{C}$
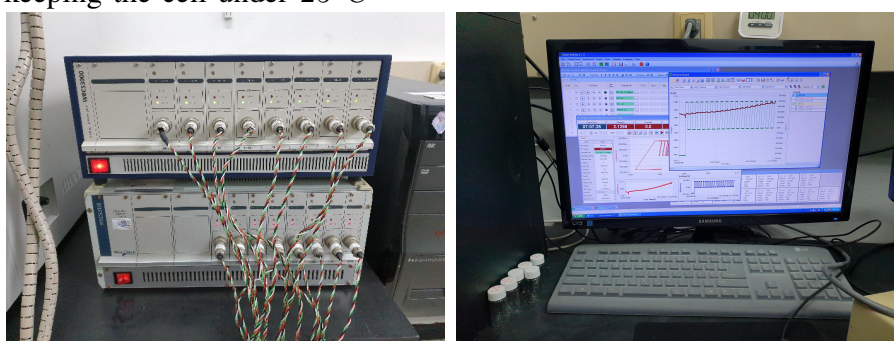

(b) The WBCS-3000s cycler(left) for galvanostatic cell test and the cell test monitoring computer system.

Fig. 9: Test bench

Model name of charge and discharge cycler is WBCS 3000s from Won-A Tech (S. Korea). The control voltage range is $\pm 5 \mathrm{~V}$ and the maximum current is $1 \mathrm{~A}$ per each channel. The test equipments are shown in Fig.9ab. The charge and discharge curves (Fig.10) are obtained by $C / 30$ current test. All tests are conducted in $25^{\circ} \mathrm{C}$ temperature. For dynamic tests, we exercised the dynamic current profile (shown partially

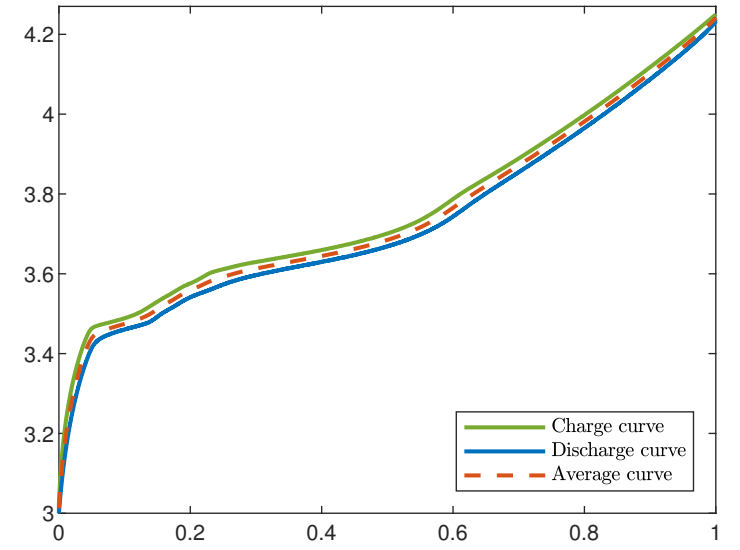

Fig. 10: $S O C-O C V$ relation at $T=25^{\circ} \mathrm{C}$.

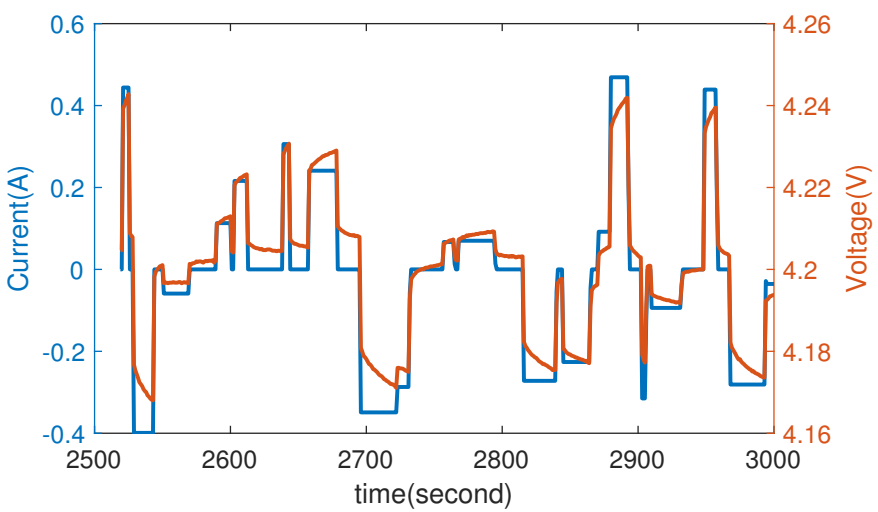

Fig. 11: Current and voltage (magnified).

in Fig.11) 40 times starting from the initial $S O C$ value of 1 , and the last $S O C$ value is 0.68 . This dynamic current profile is different from the UDDS profile considered in previous section. As we see from Fig.11, it consists of short constant current intervals.

The estimated hysteresis parameters $\widehat{M_{0}}, \widehat{M}$ and $\widehat{\eta}$ for different $S O C$ values are tabulated in Table.IV and $M$ and $M_{0}$ linearly estimated parameters for $\eta=6.5411$, which is the average value of $\widehat{\eta}$. We see that the parameter $M$ is extracted appropriately. However, instantaneous hysteresis parameter $M_{0}$ is estimated with very small negative values. This tells us instantaneous hysteresis has some small reverse effect for constant current profile, if we compare it to the previous section, where the considered UDDS current profile has many sudden jumps. Consequently, it says instantaneous hysteresis could have different characteristics depending on the current history. Nevertheless, it's effect on overall $S O C$ estimation is very little and some comparisons were made in [19]. In Fig.12, the extracted parameters $R_{0}, R, C, a, b$ and window sizes with excitation level 53 are graphed against the number of window. The variability of $R_{0}$ and $R$ are clearly shown, while $C$ takes the average value of 434 most of the times. In Fig.13, the estimated $O C V$ (purple) and the terminal voltage $V$ (light blue) are graphed for the same time interval as in Fig.11. Compared the magnitudes of $O C V$ and $V$, it is seen that the dynamics of electrical circuit model explains much part of the terminal voltage. Ideally, if $S O C$ - 
TABLE IV: Offline estimation of $M_{0}, M$ and $\eta$.

\begin{tabular}{|c|c|c|c|c|c|}
\hline$S O C$ & $M$ & $M_{0}$ & $\widehat{M}$ & $\widehat{M_{0}}$ & $\widehat{\eta}$ \\
\hline 0.964 & 0.026 & $-2.5 \mathrm{e}-05$ & 0.032 & -0.00015 & 14.7 \\
\hline 0.956 & 0.034 & $-3.3 \mathrm{e}-05$ & 0.039 & -0.00014 & 12.0 \\
\hline 0.949 & 0.042 & $-4.0 \mathrm{e}-05$ & 0.045 & -0.00015 & 11.9 \\
\hline 0.942 & 0.049 & $-6.9 \mathrm{e}-05$ & 0.051 & -0.00015 & 10.1 \\
\hline 0.935 & 0.057 & $-5.9 \mathrm{e}-05$ & 0.058 & -0.00012 & 9.1 \\
\hline 0.928 & 0.063 & $-9.4 \mathrm{e}-05$ & 0.064 & -0.00014 & 8.1 \\
\hline 0.921 & 0.071 & -0.00014 & 0.072 & -0.00017 & 7.4 \\
\hline 0.914 & 0.074 & -0.00016 & 0.074 & -0.00019 & 7.3 \\
\hline 0.907 & 0.079 & -0.00014 & 0.079 & -0.00017 & 7.1 \\
\hline 0.899 & 0.087 & -0.00015 & 0.087 & -0.00016 & 6.7 \\
\hline 0.892 & 0.092 & -0.00020 & 0.092 & -0.00019 & 6.2 \\
\hline 0.885 & 0.099 & -0.00022 & 0.099 & -0.00019 & 5.8 \\
\hline 0.878 & 0.102 & -0.00023 & 0.102 & -0.00020 & 5.8 \\
\hline 0.871 & 0.107 & -0.00020 & 0.107 & -0.00017 & 5.9 \\
\hline 0.864 & 0.115 & -0.00023 & 0.115 & -0.00018 & 5.6 \\
\hline 0.857 & 0.121 & -0.00028 & 0.121 & -0.00020 & 5.0 \\
\hline 0.850 & 0.129 & -0.00029 & 0.129 & -0.00021 & 4.9 \\
\hline 0.842 & 0.133 & -0.00031 & 0.134 & -0.00023 & 5.1 \\
\hline 0.835 & 0.140 & -0.00032 & 0.140 & -0.00023 & 4.9 \\
\hline 0.828 & 0.148 & -0.00032 & 0.148 & -0.00022 & 4.8 \\
\hline 0.821 & 0.157 & -0.00038 & 0.157 & -0.00025 & 4.4 \\
\hline 0.814 & 0.164 & -0.00041 & 0.165 & -0.00026 & 4.3 \\
\hline 0.807 & 0.166 & -0.00043 & 0.167 & -0.00029 & 4.5 \\
\hline 0.800 & 0.170 & -0.00043 & 0.171 & -0.00028 & 4.4 \\
\hline 0.793 & 0.177 & -0.00043 & 0.177 & -0.00030 & 4.7 \\
\hline 0.786 & 0.181 & -0.00049 & 0.182 & -0.00032 & 4.3 \\
\hline 0.779 & 0.187 & -0.00051 & 0.188 & -0.00033 & 4.2 \\
\hline 0.771 & 0.190 & -0.00048 & 0.190 & -0.00033 & 4.6 \\
\hline 0.764 & 0.194 & -0.00049 & 0.195 & -0.00035 & 4.8 \\
\hline 0.757 & 0.202 & -0.00051 & 0.202 & -0.00037 & 4.8 \\
\hline 0.750 & 0.208 & -0.00056 & 0.209 & -0.00039 & 4.5 \\
\hline 0.743 & 0.214 & -0.00055 & 0.215 & -0.00037 & 4.5 \\
\hline 0.736 & 0.216 & -0.00055 & 0.217 & -0.00040 & 4.9 \\
\hline 0.729 & 0.221 & -0.00054 & 0.222 & -0.00041 & 5.0 \\
\hline 0.721 & 0.228 & -0.00053 & 0.228 & -0.00041 & 5.2 \\
\hline 0.714 & 0.234 & -0.00060 & 0.235 & -0.00045 & 4.8 \\
\hline 0.707 & 0.241 & -0.00059 & 0.242 & -0.00044 & 5.0 \\
\hline 0.700 & 0.245 & -0.00057 & 0.246 & -0.00045 & 5.3 \\
\hline & & & & & \\
\hline
\end{tabular}

TABLE V: RMS errors (in percents).

\begin{tabular}{lrrrr}
\hline Methods & ES-Ah & ES-LKF & ES-EKF & ES-SPKF \\
\hline RMS & $0.9606 \%$ & $0.9611 \%$ & $0.0622 \%$ & $0.0615 \%$ \\
EOB & & $2.9075 \%$ & $5.1196 \%$ & $11.4523 \%$ \\
\hline
\end{tabular}

$O C V$ relation is just one curve (that is there is no hysteresis effect), one would expect the estimated $O C V$ is smooth piecewise lines that coincides with $S O C-O C V$ curve. However, as seen in Fig.13, the identified $O C V$ values are not exactly piece-wise lines because of the hysteresis effect. Based on the estimated hysteresis parameters in Table.IV, we see this remaining voltage difference is modeled with enough accuracy by the hysteresis model (18).

Finally, the results of $S O C$ estimation are presented in Table.V. The methods ES-Ah and ES-LKF are almost equally performed as expected. The reasons behind this better performances of these methods are the battery type, temperature and uncertainty involved. The RMS of $1 \%$ is in acceptable range for some applications. We think more research is needed in this direction. The noise variances for KF-based estimations are, experimentally, found to be $\Sigma_{w}=5 e-4$ and $\Sigma_{v}=2 e-4$. The error bounds for ES-SPKF and ES-EKF are presented in Fig.14. Both algorithms perform almost equally in this

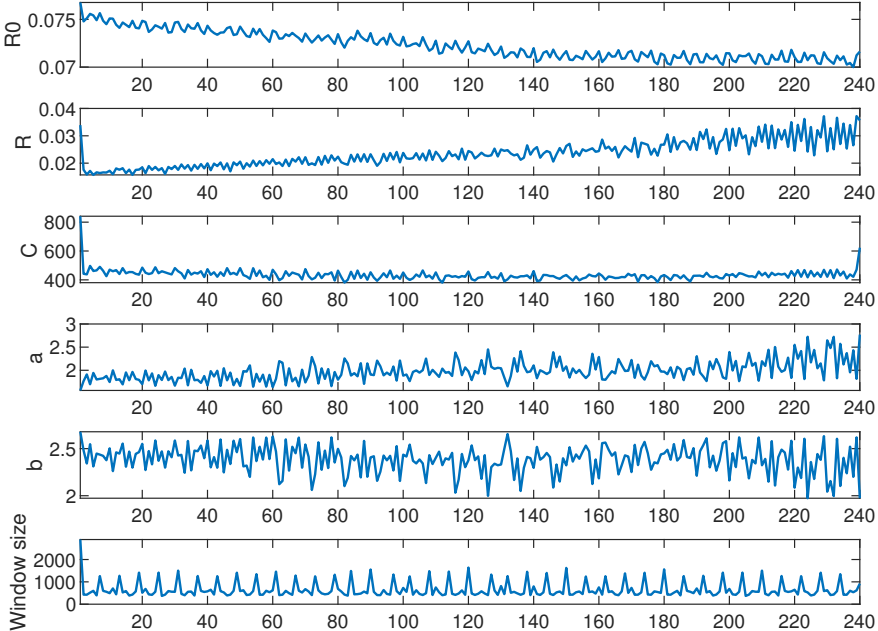

Fig. 12: Estimated parameters and time window sizes with excitation level 53.

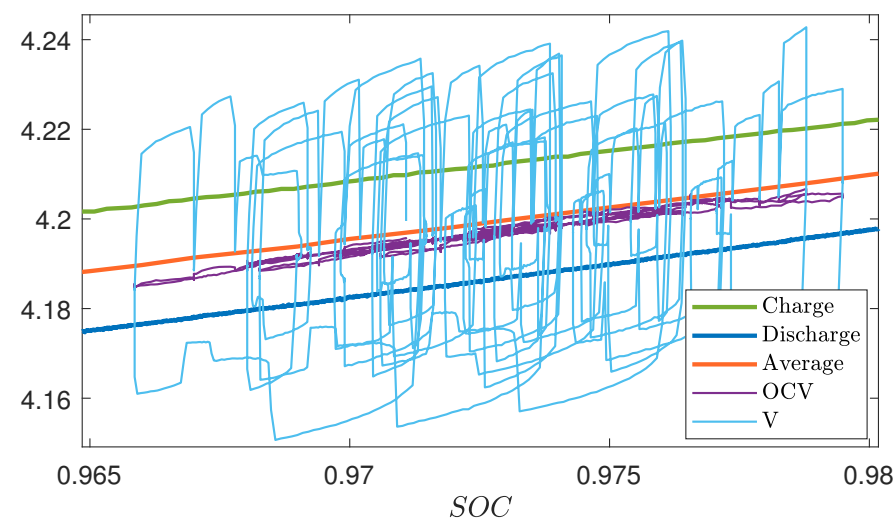

Fig. 13: The terminal voltage and the estimated $O C V$.

experiment. The error decreases to some level starting from initial uncertain $S O C$ value and uncertainty tends to grow. This is because the gap between charge and discharge curve widens to the middle and shrinks to both ends. We know the $O C V$ value depends on history of currents and we modeled the hysteresis by some displacement $\left(M, M_{0}\right)$ from the
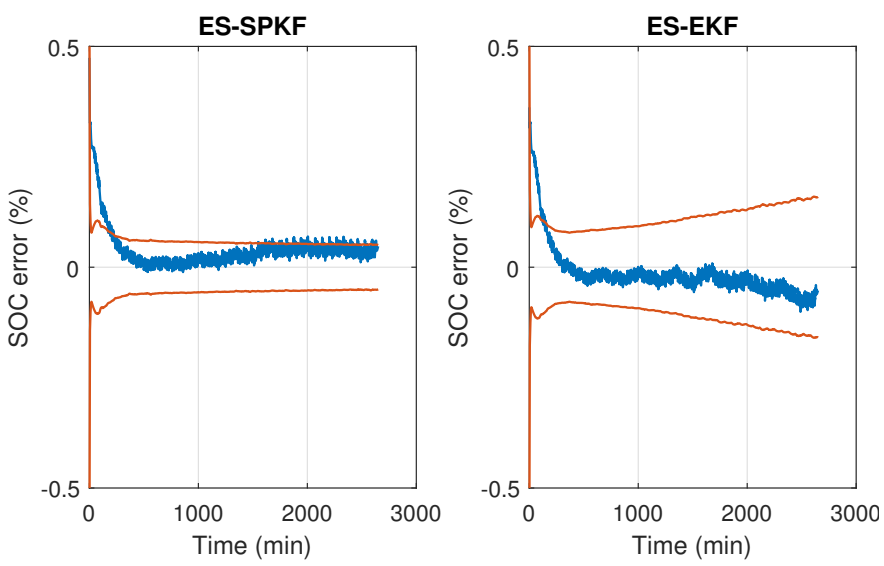

Fig. 14: Error bounds of ES-SPKF and ES-EKF estimations. 
average $O C V=f(S O C)$. Therefore, the modeling error increases to the middle of $S O C$ and decreases to both ends.

\section{CONCLUSION}

Online parameter identification and KF-based battery SOC estimation technique were proposed for an electrical battery model. Firstly, modeling the $S O C-O C V$ relation locally linear on the fly, we perform a simple linear estimation algorithm on each time window and extract battery parameters $R_{0}, R, C, a, b$ and $O C V\left[k_{0}\right]$. This procedure lets us obtain values of $O C V$. In the next phase, $\mathrm{KF}$ algorithm is executed to estimate the $S O C$ with fewer state equations. Extensive experimental tests are conducted for the real data obtained in the designated labs. The KF-based methods with hysteresis parameters estimated with $S O C$ dependence perform better than the other methods considered previously. Besides $S O C$, an important indicator of the battery's performance, which is also among the states BMS monitors, is the state of health $(S O H) . S O H$, in some sense, can be defined by the increase of resistance $R_{0}$ and the decrease of total capacity $Q$. While the resistance $R_{0}$ can be estimated online as in the section II, the estimation of $Q$ is not straightforward and requires more effort. In the next phase of this research, we plan to devise an algorithm to estimate $\mathrm{SOH}$ based on the special discretization considered in this work.

\section{ACKNOWLEDGEMENTS}

Funding: This work was supported by the National Research Fund with numbers 2017R1E1A1A03070061 and 2020R1I1A3071769.

\section{REFERENCES}

[1] K. S. Ng, C.-S. Moo, Y.-P. Chen, and Y.-C. Hsieh, "Enhanced coulomb counting method for estimating state-of-charge and stateof-health of lithium-ion batteries," Applied Energy, vol. 86, DOI https://doi.org/10.1016/j.apenergy.2008.11.021, no. 9, pp. 1506 - 1511, 2009. [Online]. Available: http://www.sciencedirect.com/science/article/ pii/S0306261908003061

[2] F. Codeca, S. M. Savaresi, and V. Manzoni, "The mix estimation algorithm for battery state-of-charge estimator- analysis of the sensitivity to measurement errors." in Proceedings of the 48h IEEE Conference on Decision and Control (CDC) held jointly with 2009 28th Chinese Control Conference, DOI 10.1109/CDC.2009.5399759, pp. 8083-8088, Dec. 2009.

[3] M. Coleman, C. K. Lee, C. Zhu, and W. G. Hurley, "State-ofcharge determination from emf voltage estimation: Using impedance, terminal voltage, and current for lead-acid and lithium-ion batteries," IEEE Transactions on Industrial Electronics, vol. 54, DOI 10.1109/TIE.2007.899926, no. 5, pp. 2550-2557, Oct. 2007.

[4] M. Chen and G. A. Rincon-Mora, "Accurate electrical battery model capable of predicting runtime and i-v performance," IEEE Transactions on Energy Conversion, vol. 21, DOI 10.1109/TEC.2006.874229, no. 2, pp. 504-511, Jun. 2006.

[7] W. Waag, C. Fleischer, and D. U. Sauer, "Critical review of the methods for monitoring of lithium-ion batteries in electric and hybrid vehicles," Journal of Power Sources, vol. 258 , DOI https://doi.org/10.1016/j.jpowsour.2014.02.064, pp. 321 - 339, 2014. [Online]. Available: http://www.sciencedirect.com/science/article/ pii/S0378775314002572
[5] R. Jackey, M. Saginaw, P. Sanghvi, J. Gazzarri, T. Huria, and M. Ceraolo, "Battery model parameter estimation using a layered technique: An example using a lithium iron phosphate cell," in $S A E$ Technical Paper, DOI 10.4271/2013-01-1547. SAE International, 04 2013. [Online]. Available: https://doi.org/10.4271/2013-01-1547

[6] R. A. Jackey, G. L. Plett, and M. J. Klein, "Parameterization of a battery simulation model using numerical optimization methods," in SAE Technical Paper, DOI 10.4271/2009-01-1381. SAE International, 04 2009. [Online]. Available: https://doi.org/10.4271/2009-01-1381

[8] C. Zhang, K. Li, S. Mcloone, and Z. Yang, "Battery modelling methods for electric vehicles - a review," in 2014 European Control Conference (ECC), DOI 10.1109/ECC.2014.6862541, pp. 2673-2678, 2014.

[9] M. U. Cuma and T. Koroglu, "A comprehensive review on estimation strategies used in hybrid and battery electric vehicles," Renewable and Sustainable Energy Reviews, vol. 42, DOI https://doi.org/10.1016/j.rser.2014.10.047, pp. 517 - 531, 2015 [Online]. Available: http://www.sciencedirect.com/science/article/pii/ S1364032114008727

[10] G. L. Plett, "Extended kalman filtering for battery management systems of lipb-based hev battery packs: Part 1-3." Journal of Power Sources, vol. 134, DOI https://doi.org/10.1016/j.jpowsour.2004.02.031, no. 2, pp. 252 - 292, 2004. [Online]. Available: http://www.sciencedirect.com/ science/article/pii/S0378775304003593

[11] G. Plett, Battery Management Systems, Volume II: Equivalent-Circuit models, ser. Artech House Power Engineering series. Artech House, 2016. [Online]. Available: http://mocha-java.uccs.edu/BMS1/index.html

[12] H. He, R. Xiong, X. Zhang, F. Sun, and J. Fan, "State-of-charge estimation of the lithium-ion battery using an adaptive extended kalman filter based on an improved thevenin model," IEEE Transactions on Vehicular Technology, vol. 60, DOI 10.1109/TVT.2011.2132812, no. 4, pp. 1461-1469, May. 2011

[13] X. Guo, L. Kang, Y. Yao, Z. Huang, and W. Li, "Joint estimation of the electric vehicle power battery state of charge based on the least squares method and the kalman filter algorithm," Energies, vol. 9, DOI 10.3390/en9020100, no. 2, 2016. [Online]. Available: http://www.mdpi.com/1996-1073/9/2/100

[14] H. Rahimi-Eichi, F. Baronti, and M. . Chow, "Modeling and online parameter identification of li-polymer battery cells for soc estimation," in 2012 IEEE International Symposium on Industrial Electronics, DOI 10.1109/ISIE.2012.6237284, pp. 1336-1341, May. 2012.

[15] H. Rahimi-Eichi and M. Chow, "Adaptive online battery parameters/soc/capacity co-estimation," in 2013 IEEE Transportation Electrification Conference and Expo (ITEC), DOI 10.1109/ITEC.2013.6574502, pp. 1-6, Jun. 2013.

[16] H. Rahimi-Eichi, F. Baronti, and M. Chow, "Online adaptive parameter identification and state-of-charge coestimation for lithium-polymer battery cells," IEEE Transactions on Industrial Electronics, vol. 61, DOI 10.1109/TIE.2013.2263774, no. 4, pp. 2053-2061, Apr. 2014.

[17] H. Rahimi-Eichi and M. Chow, "Adaptive parameter identification and state-of-charge estimation of lithium-ion batteries," in IECON 2012 38th Annual Conference on IEEE Industrial Electronics Society, DOI 10.1109/IECON.2012.6389248, pp. 4012-4017, Oct. 2012.

[18] F. Baronti, W. Zamboni, N. Femia, H. Rahimi-Eichi, R. Roncella, S. Rosi, R. Saletti, and M. . Chow, "Parameter identification of li-po batteries in electric vehicles: A comparative study," in 2013 IEEE International Symposium on Industrial Electronics, DOI 10.1109/ISIE.2013.6563887, pp. 1-7, May. 2013.

[19] M. Kwak, B. Lkhagvasuren, J. Park, and J. You, "Parameter identification and soc estimation of a battery under the hysteresis effect," IEEE Transactions on Industrial Electronics, vol. 67, no. 11, pp. 9758-9767, 2020.

[20] G. Plett, Battery Management Systems, Volume I: Battery Modeling, ser. Artech House Power Engineering series. Artech House, 2015. [Online]. Available: http://mocha-java.uccs.edu/BMS1/index.html

[21] J. L. Z. Cheng, L. Wang and J. Lv, "Estimation of state of charge of lithium-ion battery based on photovoltaic generation energy storage system.” Tehnicki vjesnik, vol. 23, DOI https://doi.org/10.17559/TV20150314130830, no. 3, pp. 695-700, 2016. 